\title{
The influence of the acoustic context on vertical sound localization in the median plane
}

\author{
STEPHAN GETZMANN \\ Ruhr-Universität Bochum, Bochum, Germany
}

\begin{abstract}
The influence of background sounds (frames) on verticallocalization of single sound sources (targets) was examined in four experiments. Loudspeakers (five targets and four frames) were positioned in the median plane, ranging from $+30^{\circ}$ to $-30^{\circ}$ above and below the subject's ear level. The subjects determined the vertical position of the targets by either verbal judgments or manual pointing. Frame and target sounds were presented concurrently or successively with a 1-sec interval; both consisted of (1) $300-\mathrm{Hz}$ square waves, (2) noise, or (3) targets of noise and frames of $300-\mathrm{Hz}$ square waves. Particularly in the second condition, the subjects consistently shifted the apparent target positions away from the frame locations. This contrast effect persisted even 1 sec after the offset of the frames. No effect was found with different waveforms for the frame and the target. Results are related to recent findings indicating a similar effect in the azimuthal dimension. Possibly the effect is based on a mechanism in which the auditory system adapts to recently heard sound source positions.
\end{abstract}

The basis of human sound localization has been studied extensively in the past (for reviews, see Blauert, 1997; Grantham, 1995; Wightman \& Kistler, 1993). It is generally accepted that the major sources of information for sound localization in the horizontal plane are interaural temporal and level differences (ITDs and ILDs). In the vertical plane, pinna-based spectral cues are crucial for judgments of sound source elevations (e.g., Butler, 1999; Hebrank \& Wright, 1974). The listeners' outer ears act as a filter that systematically modifies the frequency spectrum of incoming sound waves. Because the filtering, or the socalled head-related transfer function (HRTF), strongly depends on the elevation and the azimuth of the incoming sound, the modified proximal spectrum (measured near the eardrum) provides spectral cues that serve as a basis for vertical sound localization. In order to extract this information, it is necessary to decompose the influence of the filtering and the initial (distal) sound spectrum. Because the distal spectrum is generally unknown, it is supposed that the auditory system makes certain a priori assumptions. Sounds with relatively broad, flat, and smoothly varying spectra are reliably localized in the vertical plane (Butler \& Planert, 1976; Macpherson, 1996; Middlebrooks, 1992; Rakerd, Hartmann, \& McCaskey, 1999).

In everyday life, this physical information is supplemented by nonacoustic factors that can also influence ap-

\footnotetext{
Parts of this work were presented at the 28th Annual German Conference on Acoustics DAGA'02 in Bochum, Germany in March 2002. The author thanks Rainer Guski for his support and for critical discussion of the results and Joachim Hohnsbein and three anonymous reviewers for their very helpful comments on earlier versions of the manuscript. Correspondence concerning this article should be addressed to S. Getzmann, Ruhr-Universität Bochum, Fakultät für Psychologie, Kognitionsund Umweltpsychologie, Universitätsstr. 150, D-44780 Bochum, Germany (e-mail: stephan.getzmann@ ruhr-uni-bochum.de).
}

parent sound positions. Besides visual cues and the listener's familiarity with the sound characteristics, Wightman and Kistler (1993, p. 172) differentiated between the stimulus and the response contexts in which sound localization takes place. There are various examples for the influence of the response context. Butler and Humanski (1992) found that the apparent sound position strongly depends on the arrangement of other sound sources in space. Perrett and Noble (1995) demonstrated that "altering the constraints on response choices can have critical influences on the measurements obtained in localizationtasks" (p. 156). Furthermore, the stimulus context can play a crucial role. For instance, Good, Gilkey, and Ball (1997) showed that the presence of a masking noise may affect the accuracy of sound localization in a complex manner, depending on the signal-to-noise ratio and the masker's position relative to the subjects.

Bridgeman, Aiken, Allen, and Maresh (1997) reported a systematic shift in the apparent sound position that was caused by a background sound. They tried to transfer a visual direction illusion, the Roelofs effect (Roelofs, 1935), to the auditory modality. In the visual illusion, a small target is arranged inside a large rectangular frame. When the frame is presented with an offset from straight ahead, the position of the target appears to be shifted in the direction opposite to the frame's offset (for details, see Bridgeman, 1992; Bridgeman, Lewis, Heit, \& Nagle, 1979; Bridgeman, Peery, \& Anand, 1997). Bridgeman, Aiken, et al. replaced the visual target with an auditory target that consisted of a $300-\mathrm{Hz}$ square wave emitted from a loudspeaker in front of the subjects. The loudspeaker was shifted between trials horizontally over a $10-\mathrm{cm}$ range. The target sound was embedded in a frame sound that also consisted of a $300-\mathrm{Hz}$ square wave. The frame was emitted from an array of six loudspeakers positioned at $30^{\circ}$ to the left or the right of the center. The results were clear-cut: Targets appeared 
to be shifted in the direction opposite to the frames. These shifts were demonstrated by a sensorimotor method (pointing to the target) and by a cognitive method of localization (verbal position estimation). In a second experiment, Bridgeman, Aiken, et al. presented dissimilar sounds as targets and frames. The target was now a $400-\mathrm{Hz}$ square wave, and the frame was a $13 / \mathrm{sec}$ pulse train. No frame-dependent shifts in target localization were found in that condition.

A possibly corresponding effect, which had been reported already in the 1920s (Bartlett \& Mark, 1922; Flügel, 1921), was recently investigated further by Kashino and Nishida (1998). The lateralization of a test tone with variable ITDs was measured following exposure to a prior adapter tone. As a result, the test tone appeared to be shifted away from the adapter tone. The magnitude of this lateralization aftereffect was largest with similar frequencies of test and adapter tones and decreased with increasing frequency difference. Furthermore, the aftereffect depended on the relation of the ITDs of the test and adapter tones: The shifts reached maximum values when the ITDs differed by approximately $250 \mu \mathrm{sec}$ and were smallest when ITDs of the test and the adapter tones were similar. In addition, Kashino (1999) varied the timing of the onsets of test and adapter sounds. He also found a shift in perceived lateralization particularly when the test tone was presented concurrently with the adapter tone. With respect to the former study, Kashino (1999) assumed that "the localization aftereffect may be thought of as a special case of the simultaneous contrast effect" (p. 4).

Recently, Carlile, Hyams, and Delaney (2001) found a systematic displacement of the perceived location of a target following prolonged exposure to broadband noise also in free-field conditions. The adapter noise was presented either in front of the subjects or at $30^{\circ}$ to the right of the median plane (MP). In both cases, the displacements could be described as shifts of the targets radially away from the adapter location.

In order to explain the observed effects in horizontal sound localization, Kashino and Nishida (1998) and Kashino $(1998,1999)$ proposed a kind of spatial adaptation of the auditory system to prior sound locations. They supposed that dynamic changes in ITD-processing units occur after adaptation to a particular azimuth or, more precisely, to a particular ITD and that these temporary changes cause the localization aftereffect. In order to confirm this idea, Kashino and Nishida proposed an adaptive interaural crosscorrelation model of ITD processing that successfully simulated the psychophysical data (for details, see Kashino \& Nishida, 1998).

The aim of the present study was to investigate whether a similar contrast effect also occurs in vertical sound localization. The question of whether the perceived elevation of a sound source is also systematically influenced by background sounds is not trivial, because the mechanisms of vertical sound localization are different from those in the horizontal plane. If the contrast effect in the horizontal plane is based primarily on adaptation of ITD-processing units, a corresponding effect in the MP (where binaurally differentiated information is not available) would indicate the involvement of neural (still unknown) structures that process spectral localization cues. The analysis of stimulus context effects in vertical sound localization may provide new insights into the operation of these mechanisms.

In four experiments, the influence of a background (frame) sound on the perceived elevation of a target sound was examined. In accordance with the results of Bridgeman, Aiken, et al. (1997), a shift of the apparent target position away from the frame was expected. In addition, three issues were addressed. First, Bridgeman, Aiken, et al. reported only slight differences in the occurrence of contrast effects between a cognitive measure of localization (verbal position estimation) and a sensorimotor measure (pointing to the target). The authors concluded that a distinction between a cognitive and a sensorimotor internal representation of the auditory space does not make sense: "There is only one representation of space in the auditory mode ... that is used for both cognitive and sensorimotor functions, i.e., it controls both perceived position and acoustically guided behavior" (p. 242). If this conclusion is true, possible contrast effects in the present experiments should be similar for both manual pointing and verbal localization methods.

Second, in the study of Bridgeman, Aiken, et al. (1997) the auditory contrast effect in the horizontal plane disappeared for dissimilar target and frame waveforms, indicating that the effect did not occur due to "non-specific attentional orienting" (p. 241). Bridgeman, Aiken, et al. concluded that, in this case, "the auditory system does not use one source in helping to localize the other" (p. 242). Kashino and Nishida (1998, Experiment 1) also found a decrease in aftereffects with increasing frequency difference. Test and adapter sounds seemed to be perceived as parts of two distinct events that did not interact and that did not cause contrast effects. In order to test whether this holds true also in the vertical dimension, in the present study frames and targets of different waveforms were presented in addition.

Finally, Kashino and Nishida (1998, Experiment 2) reported that the aftereffect was strongest for test and adapter tones that differed in ITDs by approximately $250 \mu \mathrm{sec}$. The lateralization of a headphone-presented tone depends on ITDs (Yost, 1981; for a review, see Grantham, 1995) that is, the right ear leading in time gives the impression of an intracranial virtual sound source positioned to the right of the median plane. Two subsequent tones with different ITDs may be heard as two sound images separated by a certain spatial distance (except for the difference in perceived externalization). Accordingly, the aftereffect reported by Kashino and Nishida was largest for a medium separation, whereas it disappeared for two sounds that appeared either too close together or too far apart. In the present study, the influence of distance on the contrast effect in the vertical dimension was thus examined by presenting frames and targets with various angular separations. 


\section{EXPERIMENT 1}

\section{Method}

Subjects. Fifteen students participated in the first experiment. All the subjects ( 12 female, 3 male; mean age $=26.27$ years) reported normal hearing. None of them had previously participated in experiments on vertical auditory localization.

Apparatus and Stimuli. All the experiments were conducted in a sound-attenuated room $(3.7 \mathrm{~m}$ wide $\times 5.4 \mathrm{~m}$ long $\times 4 \mathrm{~m}$ high $)$ with a carpeted floor and baffles mounted at a height of $2.6 \mathrm{~m}$. The reverberation times T30 ranged from $0.34 \mathrm{sec}(5 \mathrm{kHz})$ to $0.78 \mathrm{sec}(200 \mathrm{~Hz})$. The A-weighted ambient background noise level was $35 \mathrm{~dB}$ SPL (sound pressure level, measured by a Brüel \& Kjaer Sound Level Meter Type 2226). As is shown in Figure 1A, the subject was seated on a chair in the center of the room with a chinrest to fix the head facing straight ahead. Both the chair and the chinrest were adjustable in height in order to facilitate a comfortable posture and to keep the ears of all the subjects at the same height. Seventeen loudspeakers (Soundcraft CX-320, 7-cm diameter) were mounted on a steel construction in front of the subject at a distance of $0.8 \mathrm{~m}$, pointing at the listener's head. ${ }^{1}$ The loudspeakers were covered by an opaque, black, acoustically transparent curtain ( $0.8 \mathrm{~m}$ wide and $1.2 \mathrm{~m}$ high), so that the listener knew neither the actual location nor the number of the loudspeakers used in the experiment. A white, $1.5-\mathrm{cm}$ wide centimeter scale was fixed to the curtain (Figure 1B). The numbers of the scale (font size, 22 points) ranged from 0 (down) to $166 \mathrm{~cm}$ (up); no further marks were on the scale. The chair and the chinrest were adjusted so that $106 \mathrm{~cm}$ on the scale was on the same level as the listener's eyes. This position was defined as $0^{\circ}$ on the vertical plane. As is shown in Figure 1C, the loudspeakers were mounted at nine different elevations, forming an I-shape. The five loudspeakers in the middle served as the targets (T1-T5), which the subject had to localize. These loudspeakers were selected on the basis of similar ef- ficiency and frequency response curves. Although the physical similarity of the loudspeakers was not perfect (efficiency varied by \pm 1 $\mathrm{dB}$ maximum; frequency responses to a pink noise test signal were the same within the range of $0.3-11.0 \mathrm{kHz}$ but varied by up to $7 \mathrm{~dB}$ in the region above $11 \mathrm{kHz}$ ), it seems unlikely that these slight differences could be used as cues for sound localization, because target sounds were presented only four times and in random order per condition.

Owing to mechanical restrictions, the separations between the loudspeakers were not exactly equal: The centers of the loudspeakers were on the levels of $125,116,106,97$, and $89 \mathrm{~cm}$ on the scale. These positions corresponded to the vertical angles of $13.4^{\circ}, 7.1^{\circ}, 0^{\circ}$, $-6.4^{\circ}$, and $-12^{\circ}$. Above and below the targets, 12 loudspeakers were mounted at four different elevations corresponding to the vertical angles of $30^{\circ}, 22.5^{\circ},-22.5^{\circ}$, and $-30^{\circ}$. These positions (F1F4) were defined as frame positions. As in the study of Bridgeman, Aiken, et al. (1997), each of the four frames consisted of 3 loudspeakers abreast with a center-to-center distance of $7.5^{\circ}$. Background sounds were emitted simultaneously by all 3 loudspeakers.

Sounds were generated digitally by CoolEdit96 with a 16-bit resolution and a sampling rate of $48 \mathrm{kHz}$. The stimuli were then digitalto-analog converted by a PC-controlled, 16-bit soundcard (Soundblaster $128 \mathrm{PCI}$ ) at a sampling rate of $48 \mathrm{kHz}$ and were amplified by an Uher stereoamplif ier UMA-2000. Additional filters were not used, because the spectral contributions of the alias signals were far beyond $24 \mathrm{kHz}$ (Nyquist frequency). The bandwidths of the stimuli were limited only by the bandwidth of the loudspeakers $(100 \mathrm{~Hz}-$ $13 \mathrm{kHz}$ ). In order to be better commensurate with the study of Bridgeman, Aiken, et al. (1997), we used the same stimuli in the first experiment: a $300-\mathrm{Hz}$ square wave with a duty cycle of 0.5 and abrupt onsets and offsets. As has been shown by Good and Gilkey (1996; see also Good et al., 1997), the accuracy of sound localization decreases when the signal-to-noise ratio is lowered, particularly
A

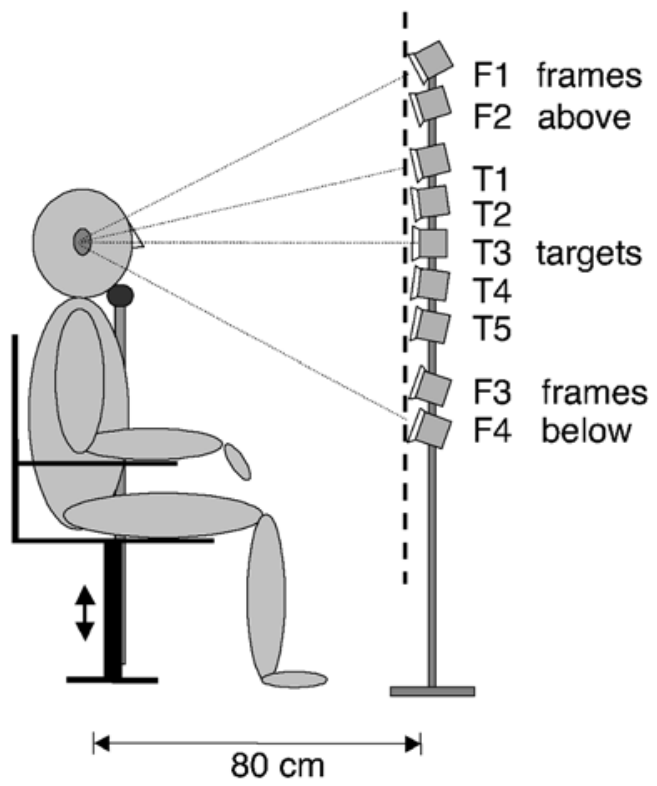

B

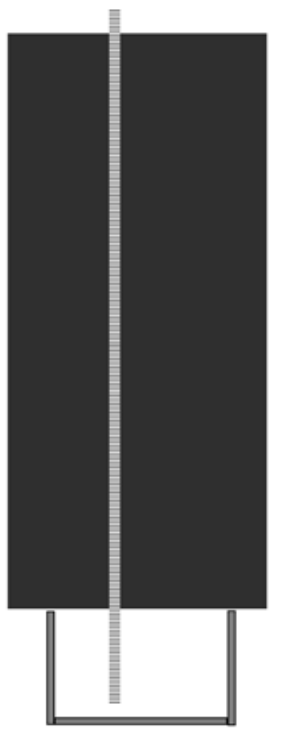

C

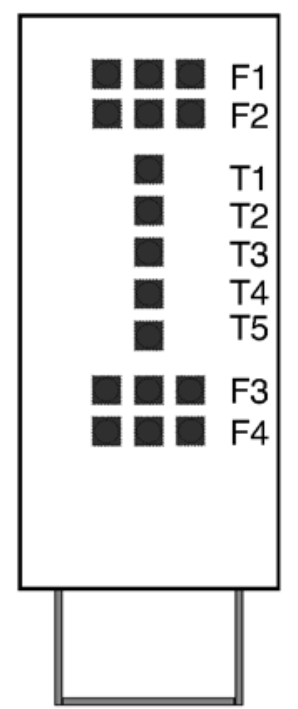

Figure 1. Schematic representation of the experimental setup. (A) Subject sitting in front of an array of loudspeakers. The positions of the upper (F1, F2) and the lower (F3, F4) frames and the targets (T1-T5) are labeled. (B) The same setup from the subjects' view. They saw only a black curtain to which a white centimeter scale was fixed. (C) The arrangement of the 17 loudspeakers forming an I-shape from the subjects' view, but without the black curtain. 
for vertical judgments. Although their results are not directly comparable with ours (signal-to-noise ratios ranged from +14 to $-13 \mathrm{~dB}$ relative to the subject's detection threshold), we found in preliminary studies that localization performance decreased when targets and frames were presented at the same intensity. The target sound used in the present study was therefore $5 \mathrm{~dB}$ louder than the frame sound [ 67 and $62 \mathrm{~dB}(\mathrm{~A})$ as measured at the listener's head position by a Brüel \& Kjaer Sound Level Meter Type 2226]. There was a constant phase shift of $230^{\circ}$ between the fundamental waves of the target and the frame square waves.

Procedure. At the beginning of each trial, the subjects kept their eyes closed. This was visually monitored by the experimenter, who could observe the subjects during the whole session. One of the four frames was activated for $2 \mathrm{sec}$. One second after the onset of the frame, one of the five targets was activated for $500 \mathrm{msec}$. Half a second after the offset of the target sound, the frame sound was also stopped, and the subjects subsequently localized the target position by either a verbal or a pointing response. In the verbal condition, the subjects opened their eyes for a moment and named the perceived position of the target relative to the centimeter scale. In the pointing condition, the subjects kept their eyes closed and pointed with the forefingers of their dominant hands to the target. The instruction was to stop the movement shortly in front of the curtain, but it was acceptable to touch the scale lightly with the fingertips. The listeners thus received no information on sizes or positions of the loudspeakers mounted shortly behind the curtain. To record the pointing response, the experimenter noted the corresponding value on the centimeter scale. In both conditions, only integer centimeter values were noted. No feedback on localization accuracy was given at any time during the experiment.

Each session began with a short practice block to familiarize the subjects with the task. The subjects were instructed to lean on the chinrest and to avoid any head movements during the sound presentations. In order to make sure that the subjects heard the targets and the frames as two distinct sounds, some targets were presented without frames. Then frames were presented individually so that the subjects could notice the differences in duration and intensity. Finally, the two sounds were presented with a delay of $1 \mathrm{sec}$ (as was described above). The subjects never reported any difficulties distinguishing between the targets and the frames. The two localization methods were practiced, and the subjects were encouraged to respond spontaneously. Then the subjects completed four experimental blocks with 40 trials each ( 80 pointing and 80 verbal) and one additional control block consisting of 20 pointing and 20 verbal trials without frames. Each stimulus combination was presented four times in pseudorandom order. The succession of the blocks changed between subjects. All five blocks were completed during a single session of approximately $1 \mathrm{~h}$.

Data analysis. First, the angular positions of the subjects' responses were calculated. In order to quantify localization performance, the following were used to compute various statistics (see also Hartmann, 1983; Lorenzi, Gatehouse, \& Lever, 1999).

1. The mean localization error, $E$, is the arithmetic mean of the differences between the subject's responses and the actual target positions. Since $E$ contains the sign of this deviation, it indicates a systematic response bias upward (positive error) or downward (negative error). The run localization error, $E^{*}$, corresponds to the error $E$ averaged across the five target loudspeakers.

2. The root-mean square (RMS) error, $D$, is the RMS average of the differences between the subject's responses and the actual target positions. We used $D$ to quantify a listener's accuracy in localizing sound sources. The run RMS error, $D^{*}$, is the RMS average across the target speakers of the RMS error $D$ for each speaker.

3. The standard deviation, $S D$, refers to the subject's responses to a stimulus produced by a target speaker. It is computed with respect to the subject's mean response and should indicate the individual localization consistency of a listener. The run $S D, S D^{*}$, is the average of $S D$ s across the target speakers.

Statistical analyses across the subjects were carried out separately for the verbal and the pointing methods. Means of the apparent target positions and mean localization errors, $E$, were plotted as functions of the actual target and frame positions. A three-factor repeatedmeasure analysis of variance (ANOVA) of the localization error data $(E)$ was carried out, assessing the main effects and interactions of target, frame, and localization method. ${ }^{2}$

\section{Results}

Figure 2 shows the apparent elevation of the targets as a function of the sound source position and the frame condition for the verbal judgment (left) and the pointing response (right). For clarity, both upper and lower frames (F1, F2 and F3, F4, respectively) are averaged in this figure.

Without frames, sound localization was quite accurate for target positions near $0^{\circ}$ elevation, but the eccentricities of

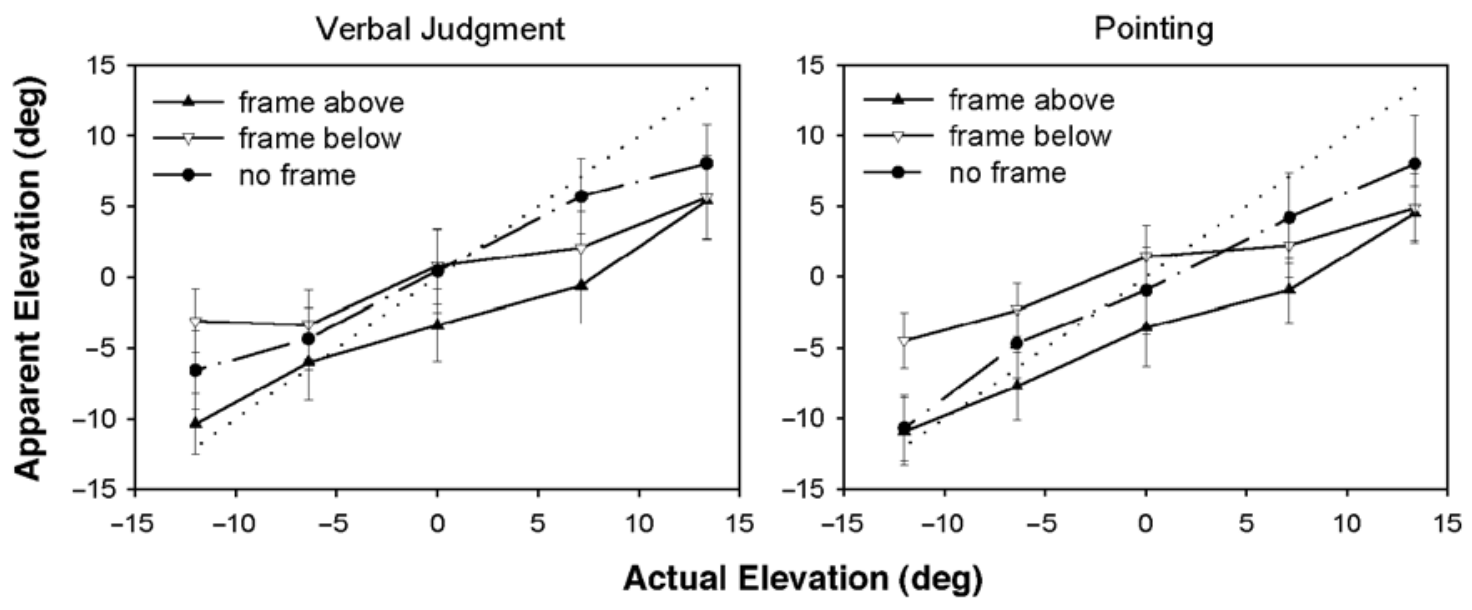

Figure 2. Apparent elevation of the target sound in Experiment 1, plotted as a function of actual source position for different frame conditions, shown separately for verbal judgments (left panel) and pointing (right panel). For clarity, both upper and lower frames $(F 1, F 2$ and $F 3, F 4$, respectively) are averaged. Data points represent mean values $( \pm S E)$ from 15 subjects. 
the peripheral targets were underestimated. In particular, the elevation of the upper target (T1) was underestimated. The ANOVA revealed the significance of the influence of the target position on the localization error $[F(4,56)=$ $6.99, p<.01]$. Run RMS errors were $D^{*}=10.33^{\circ}\left(4.81^{\circ}\right)$ for the verbal judgment and $D^{*}=10.72^{\circ}\left(5.61^{\circ}\right)$ for the pointing method (averaged across all 15 subjects with $S D \mathrm{~s}$ in parentheses). Run $S D$ s were $S D^{*}=3.73^{\circ}\left(1.83^{\circ}\right)$ and $S D^{*}=4.08^{\circ}\left(1.15^{\circ}\right)$, respectively. There were no statistically significant differences between the two localization methods. The simultaneous presentation of frame sounds in the experimental condition did not significantly change the run RMS errors. But the run standard deviation rose from $S D^{*}=3.91^{\circ}\left(1.34^{\circ}\right)$ without frames to $S D^{*}=5.24^{\circ}$ $\left(1.37^{\circ}\right)$ with frames [averaged across the four different frame positions and the two localization methods; $t(14)=$ $3.22, p<.01]$.

The frames had a systematic influence on the apparent target positions. Irrespective of the frame location, the elevations of the upper targets were underestimated. The perceived elevations of the central and the lower targets strongly depended on the frame location: They appeared to be higher for the lower frames than for the upper frames. To test whether the overall contrast effect was statistically significant, the run localization errors $\left(E^{*}\right)$ were averaged across the two upper and the two lower frames. The differences in $E^{*}$ between the upper and the lower frame conditions were $3.40^{\circ}\left(2.97^{\circ}\right)$ for the verbal method and $4.07^{\circ}$ $\left(3.68^{\circ}\right)$ for the pointing method, and both were statistically significant $[t(14)=4.44, p<.01$, and $t(14)=4.28, p<$ .01 , respectively]. There was no significant difference between the two localization methods.

Figure 3 illustrates the localization error as a function of target position and localization method for all the frame positions. When the upper frames were active (F1 and F2), a contrast effect occurred only for the upper targets and, to a lower degree, for the central target. The localization of the lower targets was slightly affected by the frames. When the lower frames were active (F3 and F4), the lower targets appeared to be shifted away from the frames, but the upper targets appeared to be shifted toward the frames. A threefactor ANOVA revealed highly significant influences of the frame position $[F(3,42)=10.49, p<.001]$ and the target position $[F(4,56)=24.84, p<.001]$ on the local-

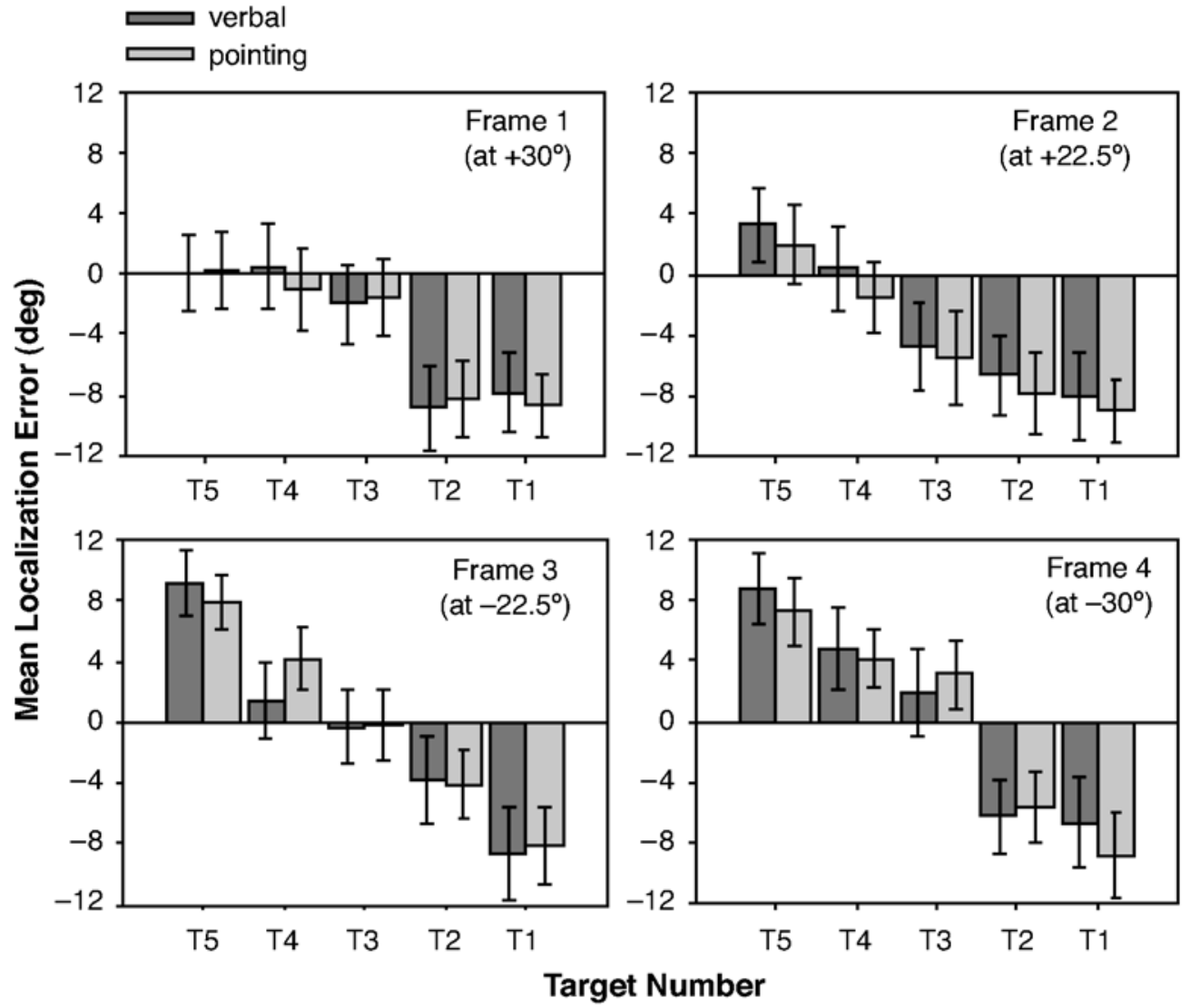

Figure 3. Mean localization error $( \pm S E)$ as a function of target position and localization method in Experiment 1 for different frame positions. Target numbers refer to the positions described in Figure 1-for example, T1 labels the upper and T5 the lower targets. Positive values indicate an overestimation of target elevation. 
ization error and a significant frame $\times$ target interaction $[F(12,168)=3.88, p<.01]$. There was no effect of localization method.

\section{Discussion}

Sound localization in the MP was systematically influenced by other sound sources. In the verbal and the pointing methods, target positions appeared to be shifted away from the frames. However, the frames influenced target localization in a highly asymmetric manner: Contrast effects occurred only for the lower and the central targets, whereas the localization of the upper targets was hardly influenced by the frame location. These targets generally appeared to be shifted downward. Thus, it seems that the magnitude of the contrast effect is diminished for targets above the ear level.

On the other hand, there were some particularities in sound localization that occurred even in the control condition - that is, when the targets were presented without frames. The subjects frequently underestimated the elevation of targets above ear level. The effect of the frames on sound localization in the experimental condition might be confounded with this general tendency. However, this cannot explain the strong asymmetry of the contrast effect, which more likely suggests a complex interaction between the target elevation and the frame location.

Biases in vertical sound localization have often been described in the literature. They occur particularly when the bandwidth of the acoustic stimulus is diminished (e.g., Butler \& Planert, 1976; King \& Oldfield, 1997; Middlebrooks, 1992) or when the stimulus spectrum is irregular (e.g., Hebrank \& Wright, 1974; Wightman \& Kistler, 1997). On the other hand, vertical sound localization is improved when spectra are relatively smooth and flat over a broad frequency range. As Middlebrooks (1992) wrote: "The brain's mechanism for sound localization must have evolved for localization of broadband sounds" (p. 2623). The spectrum of a $300-\mathrm{Hz}$ square wave such as that used in the present experiment is rich in higher harmonics, but it consists of distinct peaks. Moreover, a square wave does not have much energy in the high-frequency regions where spectral cues are used for vertical localization. Spectral cues could have been too weak to trigger a more efficient sound localization. In the second experiment, an attempt was made to reduce those possible acoustic problems, and sounds were presented that facilitate auditory localization.

\section{EXPERIMENT 2}

The influence of the auditory context on the apparent target position was tested with target and frame sounds consisting of broadband (pink) noise that may allow more precise and reliable localization in the MP.

\section{Method}

Subjects. Twelve female and 3 male students (mean age $=23.73$ years) participated in the second experiment. They had no previous experience in related experiments and reported no abnormalities in hearing.
Apparatus, Stimuli, and Procedure. The apparatus and procedure were the same as those in Experiment 1. Sounds were generated digitally by CoolEdit96 with a 16-bit resolution and a sampling rate of $48 \mathrm{kHz}$. Again, no explicit filtering was used, and the bandwidth of the stimuli was limited only by the bandwidth of the loudspeakers $(100 \mathrm{~Hz}-13 \mathrm{kHz})$. We presented pink noise (which shows a smooth decrease of higher frequencies in spectrum) rather than white noise, because it was described as less unpleasing than a white noise stimulus that we had used in a preliminary study. The frames consisted of one sample of pink noise with an abrupt onset and offset; the targets consisted of a second, independent sample. The timing of the 2-sec frame sound and the 500-msec target sound was the same as that in Experiment 1; the target sounds were again louder than the frame sounds (sound levels 65 and $60 \mathrm{~dB}[\mathrm{~A}]$, respectively).

\section{Results}

Without frames, run RMS errors were $D^{*}=7.29^{\circ}\left(2.45^{\circ}\right)$ for the verbal judgment method and $D^{*}=7.54^{\circ}\left(2.42^{\circ}\right)$ for the pointing method (averaged across all subjects, with $S D$ s in parentheses). Run standard deviations were $S D^{*}=3.84^{\circ}$ $\left(1.42^{\circ}\right)$ and $S D^{*}=3.79^{\circ}\left(1.44^{\circ}\right)$, respectively. There was no influence of the target $x$ position on the localization error, but there was a highly significant target $\times$ method interaction $[F(4,56)=6.57, p<.01]$. This may have resulted from an underestimation of the central and upper targets' elevations for the pointing method (Figure 4, right).

Frames had a consistent effect on performance (Figure 4). For the verbal and the pointing methods, the elevations of the targets were underestimated for the upper frames and overestimated for the lower frames. This contrast effect occurred particularly for the central and lower target positions, whereas it decreased for the upper target positions. As can be seen in Figure 4, the lines appear to be quite parallel below the midline and to converge above the midline. Averaged across all the target positions and the two upper and lower frame positions, the mean differences in the run localization errors between the upper and the lower frames were $7.04^{\circ}\left(5.32^{\circ}\right)$ for the verbal method $[t(14)=5.12, p<.01]$ and $6.34^{\circ}\left(5.10^{\circ}\right)$ for the pointing method $[t(14)=4.81, p<.01]$. As compared with the control condition, the simultaneous presentation of frame sounds disturbed the target localization: Averaged across the frame positions and the localization methods, the run RMS error rose from $D^{*}=7.41^{\circ}\left(2.01^{\circ}\right)$ without frames to $D^{*}=8.73^{\circ}\left(1.98^{\circ}\right)$ with frames $[t(14)=2.35, p<.05]$. The run $S D$ rose from $S D^{*}=3.81^{\circ}\left(1.33^{\circ}\right)$ to $4.72^{\circ}\left(1.15^{\circ}\right)$, respectively $[t(14)=3.81, p<.01]$.

Figure 5 shows the effect of the frame location on the localization error in detail. The central and the lower targets appeared to be shifted in the direction opposite to the frames. The upper target positions were less systematically influenced by the frames. For verbal judgments, there was a weak shift away from the active frame positions, with one exception: In the presence of the uppermost frame (F1), target T1 appeared to be shifted upward-that is, toward the frame. For the pointing method, the elevations of the upper targets were usually underestimated, which was unexpected for the lower frames. A three-factor ANOVA revealed a highly significant effect of frame position $[F(3,42)=22.16, p<.001]$ and a highly significant 


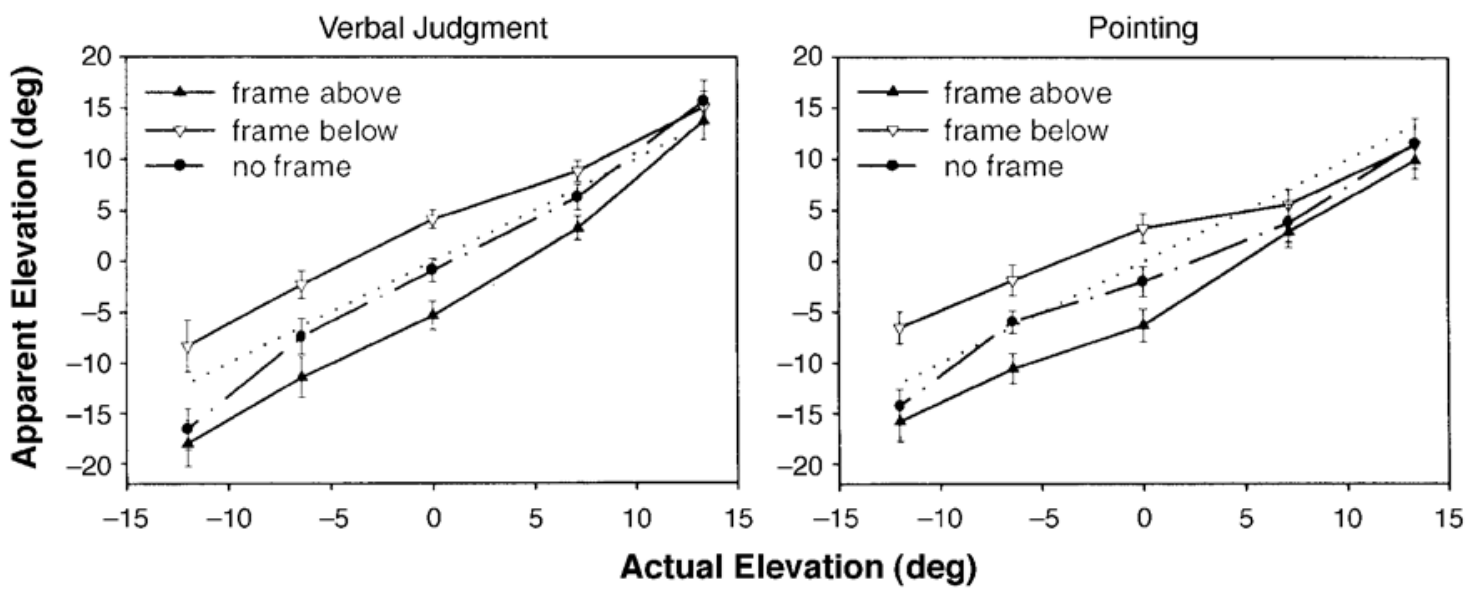

Figure 4. Apparent elevation of the target sound in Experiment 2 plotted as a function of actual source position for different frame conditions, shown separately for verbal judgments (left panel) and pointing (right panel). For clarity, both upper and lower frames $(F 1, F 2$ and $F 3, F 4$, respectively) are averaged. Data points represent mean values $( \pm S E)$ from 15 subjects.

frame $\times$ target interaction $[F(12,168)=10.12, p<.001]$ but no effect of target position or localization method. In addition, there was a significant method $\times$ target interaction $[F(4,56)=7.89, p<.01]$ on localization error.

\section{Discussion}

As was expected, the broadband noise allowed for a better sound localization than did the square wave used in Experiment 1: A comparison of the first and the second

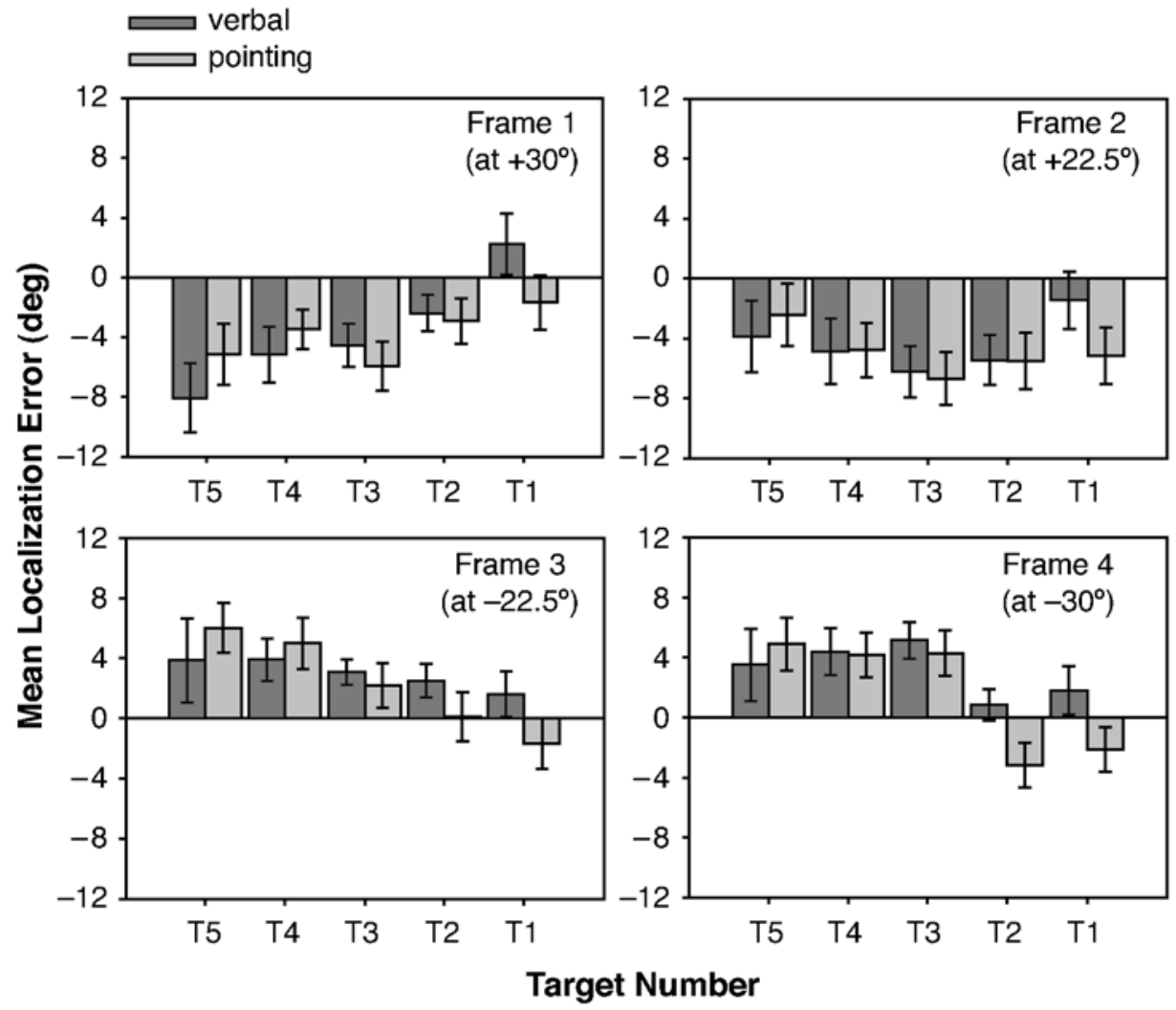

Figure 5. Mean localization error $( \pm S E)$ as a function of target position and localization method in Experiment 2 for different frame positions. Target numbers refer to the positions described in Figure 1-for example, T1 labels the upper and T5 the lower targets. Positive values indicate an overestimation of target elevation. 
experiments revealed a significant decrease in the overall run RMS error from $D^{*}=10.52^{\circ}$ to $D^{*}=7.41^{\circ}[t(18.71)=$ $2.30, p<.05 ; t$ test for independent groups].

As in Experiment 1, the target sounds appeared to be shifted away from the frame locations. However, the shift in apparent target position was more consistent than that in Experiment 1 (particularly for verbal judgments), with lower interindividual variability (cf. Figures 2 and 4). The overall contrast effect (averaged across the localization methods) was $6.69^{\circ}$, but the increase with respect to Experiment $1\left(3.73^{\circ}\right)$ was not statistically significant $[t(28)=$ $1.96, p<.1]$. Again, there was a clear effect of target elevation on the contrast effect: The central and the lower targets were much more affected than the upper targetsthat is, the contrast effect diminished for targets at upper elevations.

There were no general differences between the two localization methods; verbal judgment and pointing were comparably affected. The underestimation of the upper target elevation by pointing (and not by verbal judgment) may have resulted from our pointing method: Perhaps the effort to raise their arms induced the subjects to point rather at lower positions. This effect also occurred in the control condition and, thus, seems to be independentof specific frame influences.

\section{EXPERIMENT 3}

In the horizontal plane, Bridgeman, Aiken, et al. (1997) reported a contrast effect only for similar waveforms of frame and target. Kashino and Nishida (1998) also found that the shift in lateralization decreased for increasing frequency differences between the test and the adapter tones. In Experiments 1 and 2, the frames and the targets consisted of identical waveforms. In order to test whether the contrast effect decreases for frames and targets with different waveforms also in the MP, a third experiment was con- ducted. For reasons of better comparability, a combination of stimuli used in the former experiments was presentedthat is, a pink noise signal as target and a square wave signal as frame.

\section{Method}

Subjects. Fifteen students ( 14 female and 1 male, mean age $=26.13$ years) participated. They had no previous experience in such experiments and reported no abnormalities in hearing.

Apparatus, Stimuli, and Procedure. The apparatus and the procedure were the same as those in the former experiments. Targets consisted of pink noise; frames consisted of $300-\mathrm{Hz}$ square wave signals. Thus, both sounds were broadband signals that can generally be localized in the vertical plane, as was shown in the former experiments. Although there were some differences in the run RMS errors between the two waveforms in Experiments 1 and 2, the subjects never made extreme errors (e.g., up-down errors), which are reported when narrow band signals of different center frequencies are presented (e.g., Burlingame \& Butler, 1998; Hebrank \& Wright, 1974; King \& Oldfield, 1997; Middlebrooks, 1992; Roffler \& Butler, 1968). On the other hand, the two signals used in the present experiment have a completely different spectrum: Pink noise has a smooth sound characteristic, whereas the spectrum of a $300-\mathrm{Hz}$ square wave consists of distinct sharp peaks with a spectral spacing of $600 \mathrm{~Hz}$. Accordingly, these two stimuli sound very different. The timing of the 2-sec frame sound and the 500-msec target sound was the same as those in the former experiments. In order to improve localization performance (see above), the target sound was again of higher intensity than the frame sound [65 and $60 \mathrm{~dB}(\mathrm{~A})$, respectively].

\section{Results}

Without frame sounds, run RMS errors were $D^{*}=10.03^{\circ}$ $\left(4.66^{\circ}\right)$ for the verbal judgment and $D^{*}=8.97^{\circ}\left(3.34^{\circ}\right)$ for the pointing method. Run $S D$ s were $S D^{*}=3.88^{\circ}\left(0.95^{\circ}\right)$ and $S D^{*}=4.30^{\circ}\left(2.31^{\circ}\right)$, respectively.

In general, frames had only a weak influence on target localization. For the pointing method, there was virtually no effect of frame location on the apparent target elevation (Figure 6, right). A slight contrast shift occurred only for the central target, but not for the lower and the upper tar-

\section{Verbal Judgment}

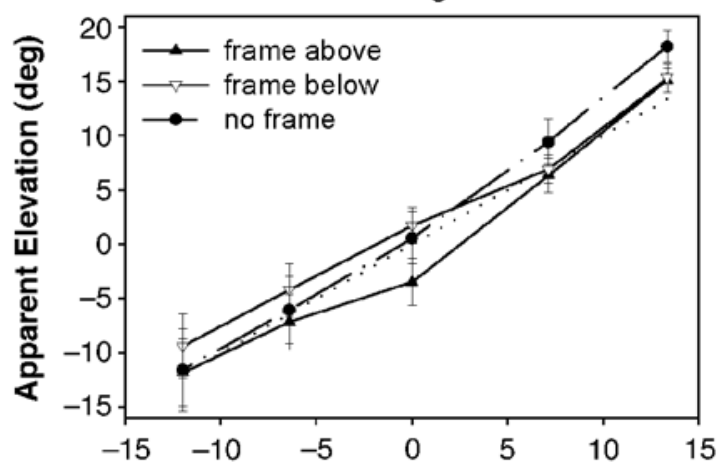

Pointing

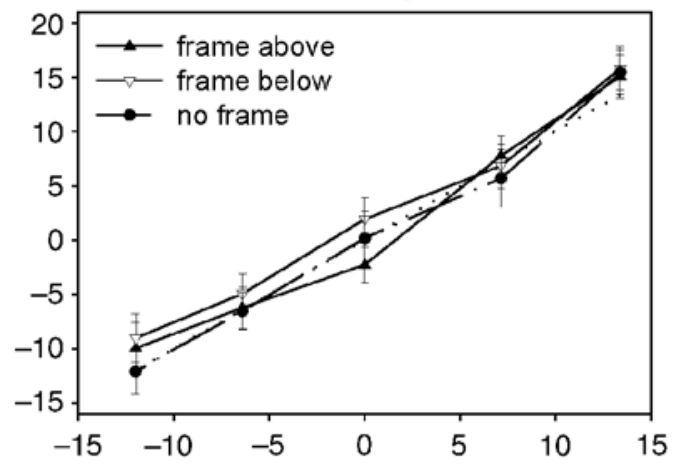

Actual Elevation (deg)

Figure 6. Apparent elevation of the target sound in Experiment 3 plotted as a function of actual source position for different frame conditions, shown separately for verbal judgments (left panel) and pointing (right panel). For clarity, both upper and lower frames $(\mathrm{F} 1, \mathrm{~F} 2$ and F3, F4, respectively) are averaged. Data points represent mean values $( \pm S E)$ from 15 subjects. 


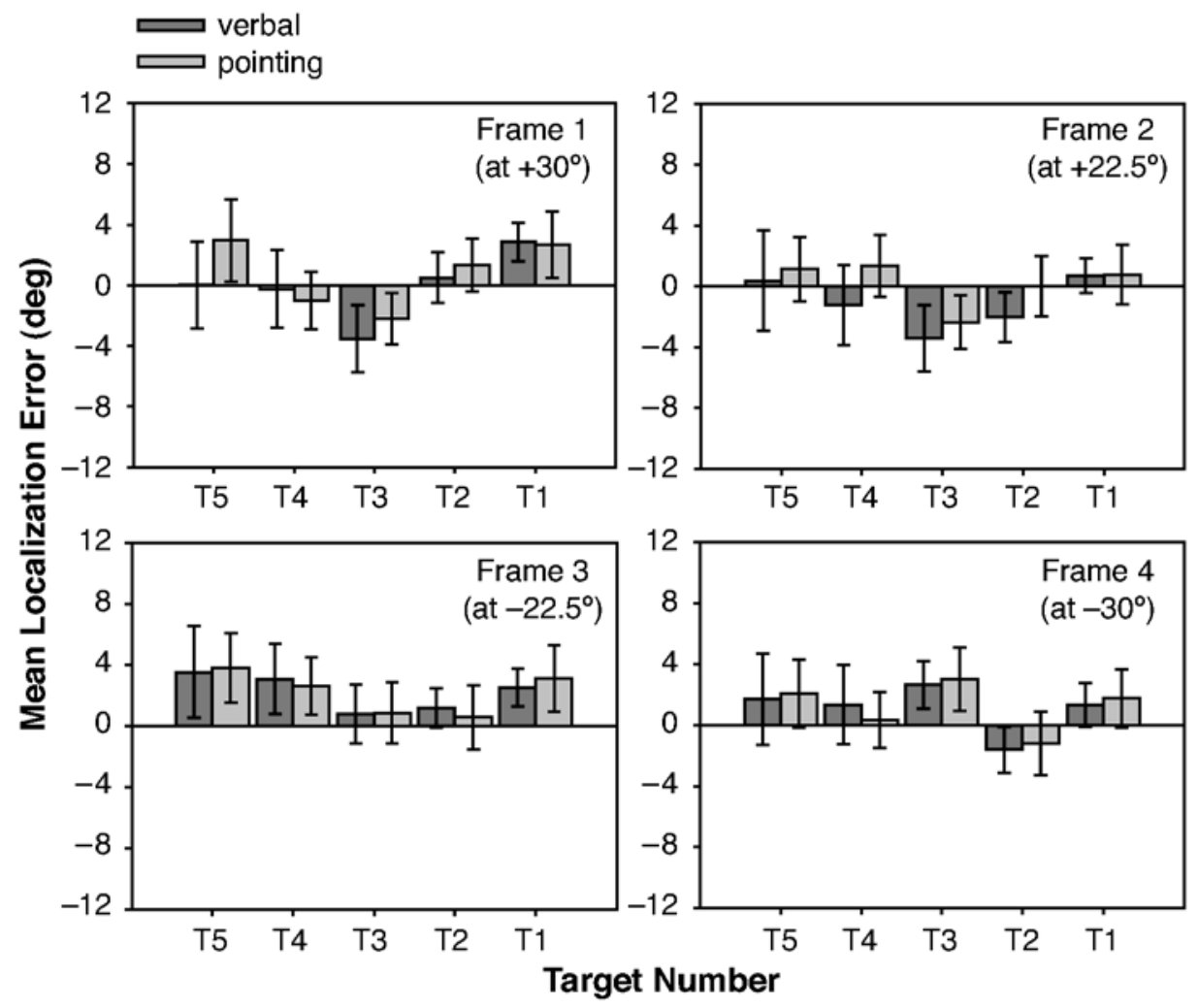

Figure 7. Mean localization error $( \pm S E)$ as a function of target position and localization method in Experiment 3 for different frame positions. Target numbers refer to the positions described in Figure 1-for example, T1 labels the upper and T5 the lower targets. Positive values indicate an overestimation of target elevation.

gets. For the verbal method, there were slight contrast shifts for the central and the lower targets (Figure 6, left). This effect vanished at higher target elevations.

Figure 7 shows in detail that a consistent contrast effect occurred only at the central target position. In order to test this effect, the mean localization errors $(E)$ was averaged across the two upper and the two lower frames. The differences between the upper and the lower frames were $5.21^{\circ}\left(4.32^{\circ}\right)$ for the verbal method and $4.22^{\circ}\left(3.79^{\circ}\right)$ for the pointing method; both were significant $[t(14)=4.67$, $p<.01$, and $t(14)=4.32, p<.01$, respectively]. For the verbal method, significant contrast effects occurred even at the lower target positions [target T4, $2.96^{\circ}\left(4.36^{\circ}\right)$, $t(14)=2.63, p<.05$; target $\mathrm{T} 5,2.43^{\circ}\left(2.81^{\circ}\right), t(14)=$ $3.35, p<.01]$, but not at the upper target positions. For the pointing method, there were no further significant contrast effects.

The overall contrast effects (averaged across all five target positions) were $2.27^{\circ}\left(2.63^{\circ}\right)$ for the verbal method and $1.25^{\circ}\left(2.15^{\circ}\right)$ for the pointing method $[t(14)=3.34, p<$ .01 , and $t(14)=2.25, p<.05$, respectively]. A three-factor ANOVA revealed a significant main effect of frame location on localization error $[F(3,42)=8.76, p<.01]$ and a frame $\times$ target interaction $[F(12,168)=7.89, p<.01]$. A weak frame $\times$ method interaction was not statistically significant $[F(3,42)=2.34, p<.1]$.

\section{Discussion}

Localization performance without frame sounds was as precise as in the second experiment. Comparisons of the RMS errors and the $S D \mathrm{~s}$ in the control conditions did not indicate significant differences. This was the result to be expected, because the same stimuli as those in Experiment 2 were used as targets.

The influence of the auditory context dramatically decreased when the target and the frame sounds were different: As compared with Experiment 2, the magnitude of the overall contrast effect significantly dropped from $6.69^{\circ}$ to $1.76^{\circ}[t(19.61)=3.52, p<.01 ; t$ test for independent groups]. But there still was a contrast effect, particularly for the verbal method and for the central loudspeaker position. In order to explain this weak interaction, a closer look at the stimuli might be useful. In Experiment 3 , we simultaneously presented a pink noise as target and a $300-\mathrm{Hz}$ square wave as frame. Although these signals differ in their sound characteristics, they overlap spectrally. The frame sound could have modified spectral cues of the target sound that are used for vertical sound lo- 
calization. Thus, there might have been an acoustic interaction. ${ }^{3}$ This possibility suggests rather an acoustic explanation for the observed effect. This issue was addressed in a fourth experiment.

\section{EXPERIMENT 4}

In trying to explain the systematic shifts in target localization, we have to consider peripheral acoustic effects. For vertical sound localization, the auditory system uses spectral characteristics of the sound-that is, frequency location and depth of spectral notches (see, e.g., Burlingame \& Butler, 1998; Carlile \& Pralong, 1994; Rakerd et al., 1999). Owing to the simultaneous presentation of two broadband sounds in Experiments 1-3, notches in the spectrum of the target sound could have been modified by the presence of the frame sound. Such a modification could systematically influence the perceived target elevation and, therefore, provide a possible explanation for the contrast effect.

Thus, the frames and the targets in Experiment 4 were presented successively. In consideration of the reflective properties of the test room (see Experiment 1), the target sound was presented $1 \mathrm{sec}$ after the offset of the frame sound. If the contrast effect observed in the former experiments arose from a purely acoustic interaction between the frame and the target sounds, no effect should occur under these conditions. In contrast, if one assumes that the effect was based on auditory adaptation, a systematic localization aftereffect should occur analogous to that reported formerly (e.g., Carlile et al., 2001; Kashino \& Nishida, 1998).

\section{Method}

Subjects. Ten students ( 8 female and 2 male, mean age $=25$ years) participated in the experiment. They had no previous experience in related experiments and reported no abnormalities in hearing.

Apparatus, Stimuli, and Procedure. The apparatus and the procedure were identical to those used in the former experiments. As in
Experiment 2, pink noise was used for target and frame sounds. But target loudspeakers were now activated $1 \mathrm{sec}$ after the offset of the 2 -sec frame sound. Immediately after presentation of the 500-msec target sound, the subjects judged its elevation as described above.

\section{Results}

In the control condition, the run RMS errors were $D^{*}=$ $7.22^{\circ}\left(3.14^{\circ}\right)$ for the verbal judgment and $D^{*}=7.33^{\circ}$ $\left(3.32^{\circ}\right)$ for the pointing method. Run $S D$ s were $S D^{*}=2.93^{\circ}$ $\left(0.88^{\circ}\right)$ and $S D^{*}=3.32^{\circ}\left(1.10^{\circ}\right)$, respectively.

As is shown in Figure 8, the preceding frames had a systematic effect on the perceived target position: The targets appeared to be located at lower elevations for the upper frames than for the lower frames. The magnitude of this contrast shift was nearly the same for the central and the lower targets but was diminished for the upper targets. To test whether the overall contrast effect was significant, the run localization errors $\left(E^{*}\right)$ were averaged across the two upper and lower frames. The differences between the upper and the lower frames were $2.39^{\circ}\left(1.08^{\circ}\right)$ for the verbal method and $2.80^{\circ}\left(1.69^{\circ}\right)$ for the pointing method, and both were statistically significant $[t(9)=6.99, p<.01$, and $t(9)=5.25, p<.01$, respectively]. A three-factor ANOVA indicated a significant main effect of frame position on the localization error $[F(3,27)=26.00, p<.01]$ but no frame $\times$ target interaction.

\section{Discussion}

Frames had an effect on the perceived target elevation, even when both sounds were presented successively. However, as compared with the former experiments, the magnitude of the aftereffect was smaller. A comparison with Experiment 2 indicates that the overall contrast effect was significantly lower $\left[2.60^{\circ}\right.$ vs. $6.69^{\circ} ; t(16.29)=3.09, p<$ $.01 ; t$ test for independent groups].

This result argues against a purely acoustic explanation for the contrast effect. Because the target was presented when the frame had ceased, acoustic modifications of the

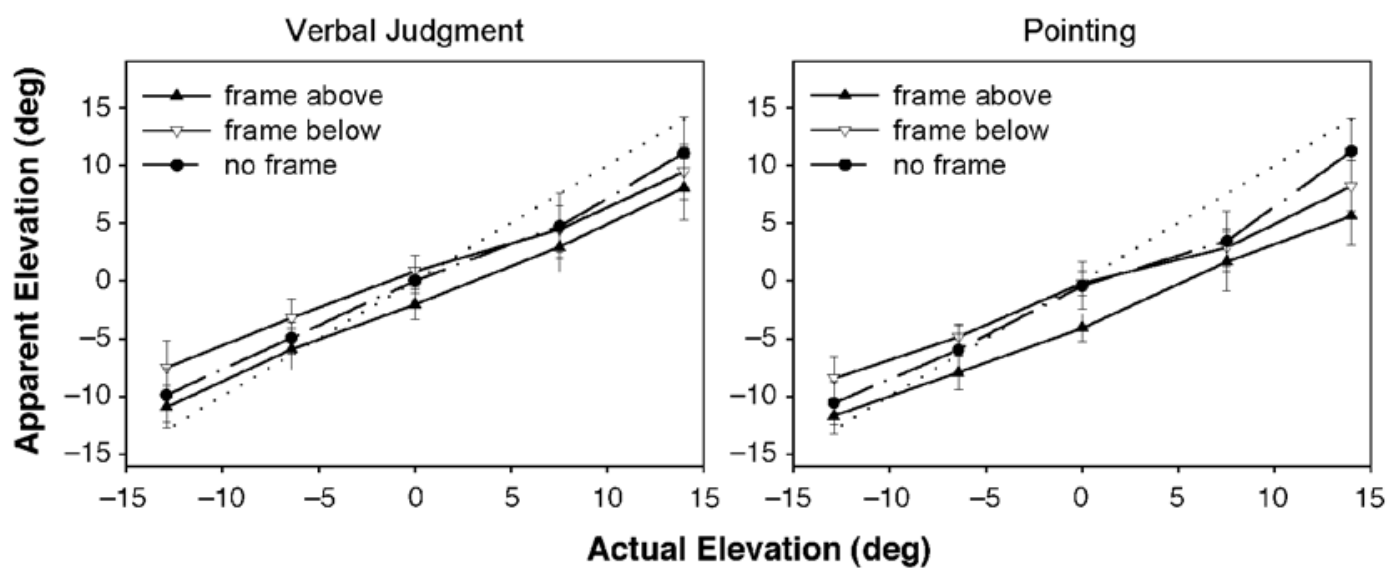

Figure 8. Apparent elevation of the target sound in Experiment 4 plotted against actual source position for different frame conditions, shown separately for verbal judgment (left) and pointing (right). For clarity, both upper and lower frames $(F 1, F 2$ and $F 3, F 4$, respectively) are averaged. Data points represent mean values $( \pm S E)$ from 10 subjects. 
target sound spectrum due to the frame sound can be excluded. Nevertheless, the targets appeared to be shifted away from the frame location, thus supporting the assumption of an auditory localization aftereffect in the processing of spectral elevation cues.

\section{GENERAL DISCUSSION}

The aim of the present study was to investigate whether the systematic influence of the acoustic context on sound localization reported by Bridgeman, Aiken, et al. (1997) for the horizontal plane also occurs in the vertical plane. It was tested whether a frame sound presented above or below ear level changed the perceived elevation of a target sound. The most important results will be summarized in the following.

Frame sounds had a systematic influence on the perceived positions of the target sounds-that is, the targets appeared to be shifted away from the frames' locations. The contrast effect was strongest in the second experiment, in which the frame and the target sounds were of identical waveforms and in which the waveforms facilitated sound localization. In these conditions, the overall displacement $\left(6.69^{\circ}\right)$ was even stronger than that reported by Bridgeman, Aiken, et al. (1997) for the horizontal plane (3.32 $)$. Several differences in the experimental settings make it difficult to compare these results quantitatively, but in any case, the data reveal the strong impact of the auditory context on sound localization.

There were only marginal differences in the results found with the two localization methods (verbal position estimation and pointing). Similar to the results reported by Bridgeman, Aiken, et al. (1997) for the horizontal plane, the auditory context may thus affect both cognitive and sensorimotor measures of sound localization. This result may support Bridgeman's conclusion that the auditory system possesses only one internal representation of space, which guides both cognitive judgments and motor behavior. ${ }^{4}$

A crucial factor for the occurrence of contrast effects is the similarity of frame and target waveforms. As was shown in Experiment 3, the impact of the auditory context strongly decreased when the frames consisted of square waves and the targets of pink noise. This waveform selectivity is consistent with results for the horizontal plane obtained by Bridgeman, Aiken, et al. (1997). They reported no significant contrast effects when the frame sound was changed to a $13 / \mathrm{sec}$ pulse train and the target sound to a $400-\mathrm{Hz}$ square wave. Kashino and Nishida (1998) also found a gradual decrease in the magnitude of the localization aftereffect for increasing differences between the test and the adapter frequencies. Bridgeman, Aiken, et al. concluded that frames and targets interact only for similar waveforms. This conclusion seems to be true also in the vertical plane, where the contrast effect was strong for similar sounds but faded away for dissimilar sounds.

When the contrast effect occurred in the simultaneous condition, the influence of the frames was stronger and more consistent for targets at central and lower elevations than for targets at upper elevations. Such an asymmetry has not been reported for the horizontal plane, where quite symmetric effects of frames on the right and the left sides on target localization have been found (Kashino 1999; Kashino \& Nishida, 1998). It may be interesting to note that the asymmetry in the vertical contrast effect decreased when the frames and the targets were presented successively: As was shown in Experiment 4, no significant frame $\times$ target interaction was found for the localization aftereffect.

In order to explain the contrast effect, we considered acoustic interactions between frame and target sounds. Thus, spectral cues for target localization might be modified by the presence of the frame sound. Some aspects of the data argue for such an explanation-for example, the fact that the contrast effect was considerably smaller when the target was presented after the frame's offset: The magnitude of the aftereffect was only about a third of the simultaneous contrast effect. This could be because those potentially important spectral interactions were unavailable in that condition. Another aspect is the strong asymmetry in the contrast effect that occurred only for the simultaneous, but not for the successive, sound presentation. Provided that the contrast effect in the simultaneous condition depends on the total received acoustic spectrum, acoustic interactions between frame and target sounds (at the eardrum) could depend on target elevation. Generally, HRTFs for sound sources above the listener tend to be rather flat (i.e., spectral localization cues are weak), whereas spectral localization cues become clearer as the source is lowered in elevation (see Middlebrooks, 1999, who derived HRTFs for different sound source elevations). 5 The interaction between frame and target sounds in the present study could be weaker for upper targets than for lower targets, resulting in a reduced contrast shift at higher elevations. Such an acoustic explanation for the asymmetry of the effects above and below ear level in the simultaneous condition may suggest that acoustic interactions could explain the contrast effect in general. However, it has not been clear until now to what extent such interactions could account for the contrast effect; the contribution of acoustics requires further exploration in future studies in which spectral differences near the eardrum should be measured for the various frame and target elevations.

The fact that there is a significant aftereffect, although any physical interaction of frame and target sounds in the nonsimultaneouscondition is impossible, suggests strongly that other mechanisms may play a role. Although we do not know what these are, one possibility is a kind of auditory spatial adaptation, as has been proposed previously for the localization aftereffect (e.g., Carlile et al., 2001; Kashino \& Nishida, 1998). Several adaptive localization models were proposed that successfully simulated the localization aftereffect. In the model of Carlile et al., the neural representation of the auditory space is formed by a map of topographically organized neurons or processing channels. Since the sensitivity of the neurons is limited to a narrow range of convergent binaural and spectral localization cues, the channels are "tuned" to different spatial 
positions. Adjacent channels substantially overlap, so that auditory spatial perception depends on the ensemble output of an array of channels. The perceived direction of a sound is determined by the zone of maximal excitation centered on particular spatial channels. (For details on the processing model and neurophysiological data providing a neuronal basis, see Carlile et al., 2001.) After auditory stimulation of an individual channel, adaptation occurs in the form of a down regulation of this channel, resulting in an imbalance of outputs from the adapted and the remaining not-stimulated channels. The crucial point is that due to the output imbalance, the zone of maximal excitation for subsequent sounds from adjacent locations shifts away from the adapted channel. Accordingly, the perceived positions of subsequent sounds shifts away from that of the preceding adapter sound. As was pointed out by Carlile et al., ". . . the down regulation of the auditory spatial channel centered on the adapting stimulus could subsequently affect the balance of outputs from the channel array for subsequent adjacent locations and result in the outward shift in the perception of nearby locations" (p. 423).

Some aspects in the present findings match this theoretical model. The frame sound started prior to the target sound and might have caused a down regulation of the corresponding processing channels. The subsequent target sound might have stimulated adjacent, not-adapted channels, so that output imbalance occurred. As a result, the apparent target elevation could have shifted away from the frame location. Assuming that the down regulation of the processing channels persists for a while, the effect should not diminish immediately after the end of the frame sound, but an aftereffect should occur. The reduced magnitude of the effect in the successive condition, in comparison with the simultaneous condition, might then be explained by a gradual recovery from adaptation. The model does not make predictions on the time course of adaptation, but the present data may suggest that adaptation occurred very quickly. A critical point is the decrease of the contrast effect for different frame and target sounds, since such a waveform selectivity is not implemented in the model. Another critical point is the spatial range in which adaptation should have an effect. The model predicts an effect for targets in the immediate vicinity of the frame sound, which decreases with increasing distance. In the present study, on the other hand, contrast effects occurred even when the frames and the targets were relatively far apart.

Altogether, it seems premature to suggest a specific neural mechanism that may make adaptation possible. However, with respect to the growing body of experimental data on the contrast effect, future models of sound localization should incorporate the influence of the auditory context.

\section{REFERENCES}

Bart Lett, F. C., \& Mark, H. (1922). A note on local fatigue in the auditory system. British Journal of Psychology, 13, 215-218.

BLAUERT, J. (1997). Spatial hearing: The psychophysics of human sound localization. Cambridge, MA: MIT Press.
BRIDGEMAN, B. (1992). Conscious vs. unconscious processes: The case of vision. Theory \& Psychology, 2, 73-88.

Bridgeman, B., Aiken, W., Allen, J., \& Maresh, T. C. (1997). Influence of acoustic context on sound localization: An auditory Roelofs effect. Psychological Research, 60, 238-243.

Bridgeman, B., Lewis, S., Heit, G., \& Nagle, M. (1979). Relation between cognitive and motor-oriented systems of visual position perception. Journal of Experimental Psychology: Human Perception \& Performance, 5, 692-700.

Bridgeman, B., Peery, S., \& Anand, S. (1997). Interaction of cognitive and sensorimotor maps of visual space. Perception \& Psychophysics, 59, 456-469.

Burlingame, J. A., \& Butler, R. A. (1998). The effects of attenuation of frequency segments on binaural localization of sound. Perception \& Psychophysics, 60, 1374-1383.

Butler, R. A. (1999). The unfolding of an auditory illusion. Perspectives in Biology \& Medicine, 42, 157-173.

Butler, R. A., \& Humanski, R. A. (1992). Localization of sound in the vertical plane with and without high-frequency spectral cues. Perception \& Psychophysics, 51, 182-186.

Butler, R. A., \& Planert, N. (1976). The influence of stimulus bandwidth on localization of sound in space. Perception \& Psychophysics, 19, 103-108.

Carlile, S., Hyams, S., \& Delaney, S. (2001). Systematic distortions of auditory space perception following prolonged exposure to broadband noise. Journal of the Acoustical Society of America, 110, 416-424.

Carlile, S., \& Pralong, D. (1994). The location-dependent nature of perceptually salient features of the human head-related transfer functions. Journal of the Acoustical Society of America, 95, 3445-3459.

Dye, R. H., Stellmack, M. A., Grange, A. N., \& Yost, W. A. (1996). The effect of distractor frequency on judgments of target laterality based on interaural delays. Journal of the Acoustical Society of America, 99, 1096-1107.

FLÜGEL, J. C. (1921). On local fatigue in the auditory system. British Journal of Psychology, 11, 105-134.

Good, M. D., \& Gilkey, R. H. (1996). Sound localization in noise: The effect of signal-to-noise ratio. Journal of the Acoustical Society of America, 99, 1108-1117.

Good, M. D., Gilkey, R. H., \& BALl, J. M. (1997). The relation between detection in noise and localization in noise in the free field. In R. H. Gilkey \& T. R. Anderson (Eds.), Binaural and spatial hearing in real and virtual environments (pp. 349-376). Hillsdale, NJ: Erlbaum.

Grantham, D. W. (1995). Spatial hearing and related phenomena. In B. C. J. Moore (Ed.), Hearing: Handbookof perception and cognition (pp. 297-345). New York: Academic Press.

Hartmann, W. N. (1983). Localization of sound in rooms. Journal of the Acoustical Society of America, 74, 1380-1391.

Hebrank, J., \& WRIGHT, D. (1974). Spectral cues in the localization of sound sources on the median plane. Journal of the Acoustical Society of America, 56, 1829-1834.

KASHINO, M. (1998). Adaptation in sound localization revealed by auditory after-effects. In A. R. Palmer, A. Rees, A. Q. Summerfield, \& R. Meddis (Eds.), Psychological and physiological advances in hearing (pp. 322-328). London: Whurr.

Kashino, M. (1999). Interaction in the perceived lateralization of two sounds having different interaural time differences. In Collected papers of the Joint Meeting "Berlin 99" (137th regular meeting of the Acoustical Society of America and the 2nd convention of the European Acoustics Association; CD-ROM). Oldenburg, Germany: DEGA e.V.

KASHINO, M., \& NISHIDA, S. (1998). Adaptation in the processing of interaural time differences revealed by the auditory localization aftereffect. Journal of the Acoustical Society of America, 103, 3597-3604.

KING, R. B., \& OlDField, S. R. (1997). The impact of signal bandwidth on auditory localization: Implications for the design of three-dimensional audio displays. Human Factors, 39, 287-295.

Lorenzi, C., Gatehouse, S., \& Lever, C. (1999). Sound localization in noise in normal-hearing listeners. Journal of the Acoustical Society of America, 105, 1810-1820.

MACPHERSON, E. A. (1996). Effects of source spectrum irregularity and 
uncertainty on sound localization [Abstract]. Journal of the Acoustical Society of America, 99, 2515.

Middebrooks, J. C. (1992). Narrow-band sound localization related to external ear acoustics. Journal of the Acoustical Society of America, 92, 2607-2624.

Middlebrooks, J. C. (1999). Individual differences in external-ear transfer functions reduced by scaling in frequency. Journal of the Acoustical Society of America, 106, 1480-1492.

Noble, W., \& Gates, A. (1985). Accuracy, latency and listener-search behavior in localization in the horizontal and vertical planes. Journal of the Acoustical Society of America, 78, 2005-2012.

Perrett, S., \& Noble, W. (1995). Available response choices affect localization of sound. Perception \& Psychophysics, 57, 150-158.

Rakerd, B., Hartmann, W. M., \& McCaskey, T. L. (1999). Identification and localization of sound sources in the median sagittal plane. Journal of the Acoustical Society of America, 106, 2812-2820.

RoElofs, C. (1935). Optische Lokalisation. Archiv für Augenheilkunde, 109, 395-415.

Roffler, S. K., \& Butler, R. A. (1968). Localization of tonal stimuli in the vertical plane. Journal of the Acoustical Society of America, 43, 1260-1266.

Shelton, B. R. \& Searle, C. L. (1980). The influence of vision on the absolute identification of sound-source position. Perception \& Psychophysics, 28, 589-596.

Wenzel, E. M., Arruda, M., Kistler, D. J., \& Wightman, F. L. (1993). Localization using nonindividualized head-related transfer functions. Journal of the Acoustical Society of America, 94, 111-123.

Wightman, F. L., \& Kist Ler, D. J. (1993). Sound localization. In W. A. Yost, A. N. Popper, \& R. R. Fay (Eds.), Human psychophysics (pp. 155192). New York: Springer-Verlag.

Wightman, F. L., \& KistLer, D. J. (1997). Monaural sound localization revisited. Journal of the Acoustical Society of America, 101, 1050-1063.

Yost, W. A. (1981). Lateral position of sinusoids presented with interaural intensive and temporal differences. Journal of the Acoustical Society of America, 70, 397-409.

\section{NOTES}

1. The distance of $0.8 \mathrm{~m}$ was chosen with regard to the localization method of finger-pointing used in the experiments (see below). To make sure that the observed data (which may reflect properties of near-field sound localization) are not due to the short loudspeaker distance, we conducted additional sessions in which we presented the sounds at a distance of $1.4 \mathrm{~m}$. The results were consistent with those of the main experiment.

2. Since there is some evidence of idiosyncratic behavior in auditory localization (e.g., Dye, Stellmack, Grange, \& Yost, 1996; Wenzel, Arruda, Kistler, \& Wightman, 1993), we additionally examined the individual localization performance of our subjects. Although there were some minor variations in all the experiments, these differences did not alter the conclusions based on averages.

3. One could speculate whether the contrast effect would completely disappear when nonoverlapping signals are used as frames and targetsfor example, low- and highpass signals. But these stimuli would be hard to localize in the vertical plane, and the absence of the contrast effect would be confounded with these difficulties.

4. An alternative explanation for the occurrence of auditory effects in pointing and verbal responses might be that the planning of the arm movement involved higher cortical systems that also guided cognitive judgments. Even if only these higher cortical "cognitive" systems were incapable of discounting the effect of the context, the contrast shift would occur in both localization measures. In further investigations, one could measure other "low-level" reactions (e.g., startle responses) to better distinguish between a cognitive and a sensorimotor representation of the auditory space. For now, one can state that there is an effect of the auditory context, but it seems premature to suggest the specific stage of representation at which it occurs.

5. Accordingly, sound localization in the MP is best for sources in the front and worst for sources that are above ear level or in the rear (e.g., Noble \& Gates, 1985; Shelton \& Searle, 1980; for a review, see Wightman \& Kistler, 1993)

(Manuscript received October 18, 2000;

revision accepted for publication March 19, 2003.) 